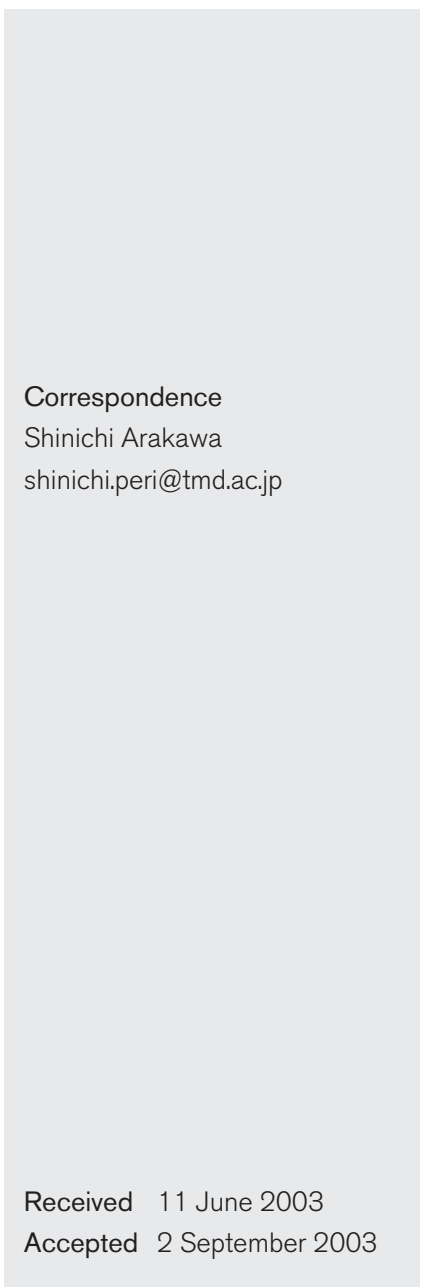

\section{Cloning of the Tannerella forsythensis (Bacteroides forsythus) siaHI gene and purification of the sialidase enzyme}

\author{
Hiroaki Ishikura, ${ }^{1,2}$ Shinichi Arakawa, ${ }^{2,3}$ Takuma Nakajima, ${ }^{2}$ \\ Nobuo Tsuchida ${ }^{2}$ and Isao Ishikawa ${ }^{1}$
}

Section of Periodontology ${ }^{1}$, Section of Molecular and Cellular Oncology and Microbiology ${ }^{2}$ and Dental Hospital, Section of Periodontology ${ }^{3}$, Tokyo Medical and Dental University, Graduate School, 1-5-45, Yushima, Bunkyo-Ku, Tokyo 113-8549, Japan

\begin{abstract}
Tannerella forsythensis (previously named Bacteroides forsythus) is a Gram-negative, anaerobic, fusiform bacterium that is a primary or secondary aetiological agent in periodontal disease in humans. T. forsythensis expresses several putative virulence factors, including a sialidase; however, there has been no molecular genetic characterization of this enzyme. A sialidase clone ( $\mathrm{pHI}-1)$ was screened from a total of 455 recombinant clones of a genomic DNA library using the 2' -

(4-methylumbelliferyl)- $\alpha$-D- $N$-acetylneuraminic acid (MUNeuAc) filter-paper spot assay. The sialidase gene ORF ( $\mathrm{siaHI}$ ) consists of a $1395 \mathrm{bp}$ coding sequence and encodes a protein with 465 amino acids with an overall molecular mass of $52 \mathrm{kDa}$. The sialidase does not have sequence similarity to any other bacterial sialidase. The entire sialidase ORF was expressed in Escherichia coli. Furthermore, the sialidase was purified from the type strain of $T$. forsythensis and from a recombinant clone, $\mathrm{pH}-1: 1$, and was analysed using a non-denaturing gel, revealing that the enzyme preparations were respectively separated as two major bands and as a single band. Southern blot hybridization analysis revealed similar patterns of siaHl-hybridizing bands among clinical isolates of T. forsythensis from periodontitis patients. This is the first study on the cloning and expression of a T. forsythensis sialidase gene and the purification of the SiaHI enzyme from T. forsythensis ATCC $43037^{\top}$ and recombinant E. coli.
\end{abstract}

\section{INTRODUCTION}

Tannerella forsythensis (Bacteroides forsythus), a Gramnegative, anaerobic, fusiform bacterium, has been implicated as one of the periodontal pathogens associated with periodontitis. Several studies have shown a strong correlation of $T$. forsythensis with the severity of periodontitis in adult patients (Grossi et al., 1994, 1995; Tanner et al., 1985, 1986). Identification of virulence factors of this bacterium could aid in the development of strategies for prevention and treatment of periodontitis. However, due to difficulties in culturing this organism from periodontitis patients, its role in the initiation and progression of periodontitis has been difficult to determine. Only a few putative virulence factors have been identified in this bacterium, based on their in vitro properties, including a sialidase, a trypsin-like protease, $N$-benzoyl-Val-Gly-Arg- $p$-nitroanilide-specific protease, a

Abbreviation: MUNeuNAc, 2'-(4-methylumbelliferyl)- $\alpha$-D-Nacetylneuraminic acid.

The GenBank/EMBL/DDBJ accession number for the siaHl gene sequence of $T$. forsythensis ATCC $43037^{\top}$ is AY069941. cell surface-associated BspA protein and a cell-deathinducing factor (Arakawa et al., 2000; Holt \& Bramanti, 1991; Saito et al., 1997; Sharma et al., 1998).

Several bacterial enzymes, including phospholipases and proteases, have been suggested as potential virulence determinants in prematurity (McGregor et al., 1990). Among these, there has recently been interest in the sialidases of pathogenic bacteria and their potential role in pathogenesis. Sialidases are enzymes that cleave $\alpha$-ketosidic linkages between sialic acid and the glycosyl residues of glycoproteins, glycolipids or colominic acids. Sialidase activity has been found in viruses, bacteria, protozoa, fungi and metazoans (Berry et al., 1988). The sialidase of the Gram-negative bacillus Erysipelothrix rhusiopathiae has been shown to be involved in infection and tissue destruction (Gyles, 1986). Anaerobic Gram-negative rods belonging to the genera Prevotella and Bacteroides were suggested to be the main sources of sialidase activity in vaginal fluid (Briselden et al., 1992). Bacteroides isolates obtained from pathological specimens were found to have significantly higher levels of sialidase activity than isolates from non-pathological specimens (Grossi et al., 1994). Sialidases have been demonstrated 
to modify a variety of host-associated activities that result in a modification of the host's ability to respond to bacterial infection and could be an important virulence factor of T. forsythensis. However, little is known about how this putative $T$. forsythensis virulence factor may contribute to its pathogenesis in vivo. In order to define the molecular nature of the $T$. forsythensis sialidase and as a first step to determining its specific role in the virulence of this bacterium, the sialidase gene was isolated, its nucleotide sequence was determined, recombinant sialidase was expressed in Escherichia coli and sialidases were purified from both T. forsythensis and the E. coli clone.

\section{METHODS}

Bacterial strains, media and growth conditions. In this study, we used T. forsythensis ATCC $43037^{\mathrm{T}}$ and three clinical isolates (42-11, 29-41 and 33-22) that were isolated in our periodontal clinic from periodontitis patients, with their consent. These bacteria were grown anaerobically in heart infusion broth (Difco) containing $5 \mu \mathrm{g}$ haemin $\mathrm{ml}^{-1}$ (Wako), $1 \mu \mathrm{g} \mathrm{ml}^{-1}$ menadione, $1 \%$ L-cysteine, $15 \mu \mathrm{g} \mathrm{N}$ acetylneuraminic acid $\mathrm{ml}^{-1}$ (Sigma) and $10 \%$ sheep blood (Nippon Bio-Test Laboratories), with or without $1.5 \%$ agar. E. coli HB101 (Takara Shuzo) containing plasmid pBluescript SKII (Stratagene) was grown in Luria-Bertani (LB) medium (Difco) with $100 \mu$ g ampicillin $\mathrm{ml}^{-1}$.

Isolation of chromosomal DNA. After culturing as described above, T. forsythensis ATCC $43037^{\mathrm{T}}$ and the clinical isolates were harvested by centrifugation $\left(5000 \mathrm{~g}\right.$ at $4{ }^{\circ} \mathrm{C}$ for $20 \mathrm{~min}$ ). Chromosomal DNA was isolated using the method of Marmur (1961).

Construction of a T. forsythensis genomic library and screening for sialidase-producing clones. Chromosomal DNA of $T$. forsythensis ATCC $43037^{\mathrm{T}}$ was isolated, size-fractionated $(4-10 \mathrm{kbp})$ using a QIAquick gel extraction kit (Qiagen) after partial digestion with Sau3AI (New England Biolabs) and ligated to the BamHI site of pBluescript SKII (+). This ligation reaction was used to transform E. coli HB101 and transformants were incubated aerobically on LB-ampicillin agar plates. Colonies were lifted from the agar plates onto a membrane (Hybond$\mathrm{N}+;$ Amersham) and transferred to another agar plate. The plates were incubated overnight at $37^{\circ} \mathrm{C}$. Colonies were transferred from this agar plate to a nylon membrane and soaked in a $100 \mu \mathrm{M}$ solution of the fluorogenic neuraminidase substrate $2^{\prime}$-(4-methylumbelliferyl)- $\alpha$-D$\mathrm{N}$-acetylneuraminic acid (MUNeuNAc sodium salt; Sigma) (Myers et al., 1980) in $0 \cdot 17 \mathrm{M}$ sodium acetate buffer ( $\mathrm{pH} 5 \cdot 4$ ) and incubated at $37^{\circ} \mathrm{C}$ for $10-15 \mathrm{~min}$. Colonies on the membrane were examined for sialidase activity by detecting the release of 4-methylumbelliferone from MUNeuNAc as fluorescence under long-wavelength UV light (450 nm) (Moncla \& Braham, 1989).

Nucleotide sequencing of $T$. forsythensis sialidase gene. Nucleotide sequencing of the sialidase-positive clone (pHI-1) was performed using the dideoxynucleotide chain termination method of Sanger et al. (1977), with a template of double-stranded plasmid DNA and sitespecific synthetic oligonucleotide primers (Nisshin Seifun Corp.). Template plasmid DNA was prepared with a QIA plasmid isolation kit (Qiagen). The sequences of both DNA strands were determined and all sequences overlapped with adjacent sequences. Nucleotide sequences were assembled and analysed using DNASIS software (Hitachi).

Construction of deletion plasmids. In order to identify the smallest fragment that encoded sialidase, deletion plasmids were constructed (see Fig. 2a) and activity was measured using a fluorescent assay method
(Myers et al., 1980). The digested fragments were separated on 1.0\% agarose gels and purified from these agarose gels using a QIAquick gel extraction kit (Qiagen). The fragments were ligated with unique sites of pBluescript SKII $(+)$ and pBluescript KSII $(+)$, digested with the appropriate enzymes. The deletion plasmids thus obtained were examined qualitatively for sialidase activity as outlined above.

In vitro mutagenesis. The predicted sialidase structural gene, containing the first ATG codon of ORF1 (translation start codon), was amplified by PCR from pHI-1. We used the following synthetic oligonucleotide primers: R1 (sense primer, 5'-CCGAATTC AAAATTGTCATGACA-3'; EcoRI site underlined), R2 (sense primer, 5'-CCGAATTCAAAATTGTCCGGACA-3'; ATG start codon changed to CGG) and F1 (antisense primer, 5'-GGCTCGAGTCAT CACTTTTTCTC-3'; XhoI site underlined) (see Fig. 2b). Pwo DNA polymerase (Roche Diagnostics), known for its high proofreading activity (Roggentin et al., 1989), was used in order to prevent unwanted mutations. After digestion with EcoRI and XhoI, these products were ligated to the appropriate site of pBluescript SKII $(+)$ and used to transform E. coli HB101. Transformants were screened for sialidase activity as outlined above.

Chromatography. The ÄKTA explorer 10S protein purification system (Amersham Pharmacia Biotech) was employed for column chromatography to regulate buffer flow and to monitor elution status and absorbance at 254 and $280 \mathrm{~nm}$ was recorded automatically. The sialidase-positive clone (pHI-1:1) and T. forsythensis were cultured in 51 broth and grown to early stationary phase. Bacterial cells were harvested by centrifugation at $5000 \mathrm{~g}$ for $30 \mathrm{~min}$ at $4{ }^{\circ} \mathrm{C}$. Cells were disrupted by sonication, centrifuged to remove cellular debris and finally filtered using a $0.22 \mu \mathrm{m}$ filter. At the first step, $15 \mathrm{ml}$ of the resultant crude extract was diluted with $35 \mathrm{ml}$ phosphate buffer $\left(10.1 \mathrm{mM} \mathrm{Na}_{2} \mathrm{HPO}_{4}, 1.76 \mathrm{mM} \mathrm{KH}_{2} \mathrm{PO}_{4}\right)$ and loaded onto a cationexchange Resource $\mathrm{S}$ column (Amersham) equilibrated with $0 \cdot 1 \mathrm{M}$ $\mathrm{MES} / \mathrm{NaOH}(\mathrm{pH} 6.0)$ buffer at a flow rate of $2 \mathrm{ml} \mathrm{min}^{-1}$. Adsorbed components were eluted with a $300 \mathrm{ml}$ linear gradient of $\mathrm{NaCl}$ from 0 to $1 \mathrm{M}$. Active fractions with sialidase activity were collected and dialysed against $50 \mathrm{mM}$ Tris/ $\mathrm{HCl}(\mathrm{pH} 8.0$ ) buffer using a Hitrap column (Amersham). Thereafter, the concentrate was subjected to an anionexchange Resource Q column (Amersham) equilibrated with $50 \mathrm{mM}$ Tris/HCl (pH 8.0) buffer. Active sialidase fractions were collected and concentrated and $\mathrm{NaCl}$ was removed using a vivaspin centrifugal concentrator (exclusion molecular mass $10 \mathrm{kDa}$; Sartorius). Protein concentrations were measured using Protein Assay reagent (Bio-Rad) as an equivalent concentration of BSA.

Non-denaturing PAGE. Since the purified preparations stagnated in the stacking gel during SDS-PAGE, the purity and activity of the purified enzymes were determined by non-denaturing PAGE, which was performed according to the method of Bhown \& Bennett (1983). All buffers and solutions were made fresh, stored at $4{ }^{\circ} \mathrm{C}$ and used within a week to obtain the most consistent results. Non-denaturing gels (10\%) were electrophoresed at $4{ }^{\circ} \mathrm{C}$ for $4-5 \mathrm{~h}$. The gel was then washed with $0 \cdot 17 \mathrm{M}$ sodium acetate buffer ( $\mathrm{pH} 5 \cdot 4$ ) for 5 min and sialidase activity was examined with the fluorogenic substrate as described above. In order to visualize sialidase activity, a CCD image of the gel was taken under long-wavelength UV light $(450 \mathrm{~nm})$ and another gel loaded under the same conditions was stained with $0 \cdot 2 \%$ Coomassie brilliant blue R-250 in $50 \%$ methanol and destained for $1 \mathrm{~h}$ (two or three times) at room temperature in order to visualize protein bands.

Southern blot analysis. DNA from clinical isolates and T. forsythensis ATCC $43037^{\mathrm{T}}$ was digested with BbsI (New England Biolabs). Following electrophoresis on $1 \%$ agarose gels, the separated DNA fragments were transferred onto a Hybond $-\mathrm{N}^{+}$membrane (Amersham) using a capillary transfer technique (Southern, 1975). Transferred DNA frag- 
ments were cross-linked to the membrane using a UV cross-linker (FUNA; Funakoshi). The 2-3-kbp BbsI-BbsI DNA fragment of pHI-1 was labelled with peroxidase using an ECL non-radioactive labelling kit (Amersham) and hybridized at high stringency to the membrane, in accordance with the manufacturer's instructions.

\section{RESULTS}

\section{Cloning of T. forsythensis ATCC $43037^{\top}$ sialidase gene}

A clone bank of T. forsythensis ATCC $43037^{\mathrm{T}}$ DNA fragments inserted in the cloning vector pBluescript SKII $(+)$ was constructed using E. coli HB101, a strain with no endogenous sialidase activity (Vimr \& Troy, 1985). Of a total of 455 colonies, one was found to be positive for sialidase activity, as indicated by results of a filter-paper spot assay. This colony was purified twice more using the single colony isolation method. It was then retested and found to be positive for sialidase activity. This clone, designated pHI-1, was used for subsequent analysis (Fig. 1).

\section{Identification of an ORF encoding sialidase}

The nucleotide sequence of each strand of the $3 \cdot 2-\mathrm{kbp}$ insert of pHI-1 was determined (Fig. 2a). Four ORFs were identified in the inserted DNA of pHI-1. In order to determine which ORF encodes sialidase, several deletion derivatives of $\mathrm{pHI}-1$ were constructed. The sialidase activity of each derivative was assessed using a filter paper spot assay

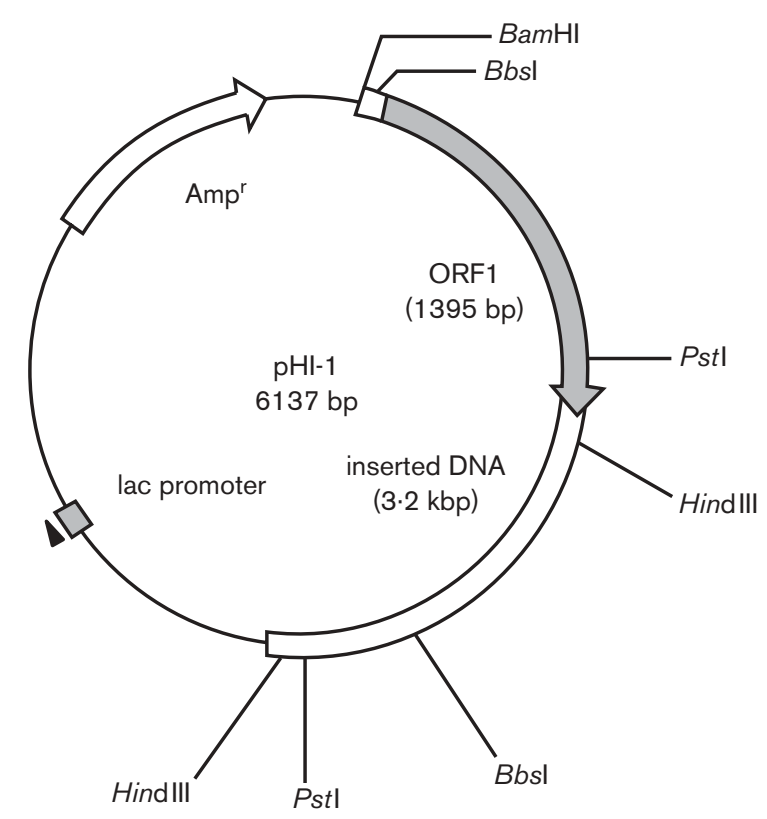

Fig. 1. Restriction map of clone $\mathrm{pH}-1$. The map shows the initiation site and direction of transcription of ORF1 ( $\mathrm{siaHI})$, as determined by sequence analysis of inserted DNA in pHI-1. The 3.2-kbp Sau3Al fragment was cloned into the unique $\mathrm{BamHI}$ site within the multiple cloning region of pBluescript SKII (+).

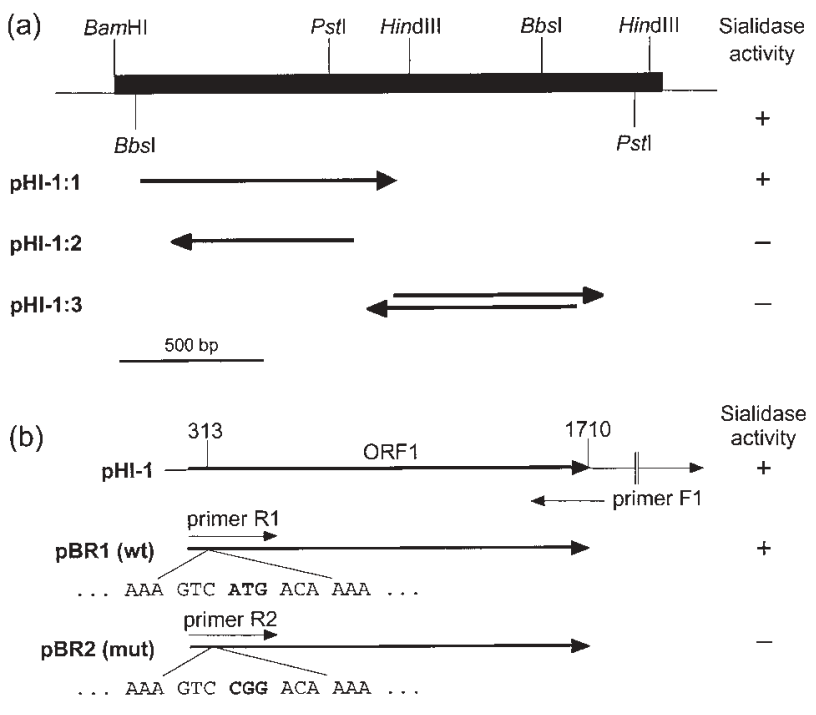

Fig. 2. (a) Construction of deletion mutants and their sialidase activity. In order to identify the smallest fragment that encoded sialidase, deletion mutants were constructed and sialidase activity was assayed using a filter-paper spot assay. Deletion mutants were made in pBluescript SKII (+) and contained the following fragments: BamHI-HindllI in the forward direction $(\mathrm{pHI}-1: 1)$ and reverse direction $(\mathrm{pHI}-1: 2) ;$ Pstl-Pstl $(\mathrm{pH}-1: 3)$. The arrow indicates the direction of transcription in each ORF. Similar results were obtained in several repetitions of this experiment. (b) In vitro mutagenesis. Arrows indicate PCR primers used to amplify the DNA and create EcoRl and Xhol sites. R2 was designed to replace the initiation codon (first ATG codon) with a CGG codon. pBR1 contained the wild-type ORF1 (primers R1 and F1) and pBR2 contained the mutant ORF (primers R2 and F1). Similar results were obtained in several repetitions of this experiment. Sequences were confirmed using an ABI 370 DNA sequencer.

(Fig. 2b). The deletion derivatives pHI-1:1 and pHI-1:2 contained the 1.4-kbp BamHI-HindIII fragment of pHI-1 in pBluescript SKII (+) and pBluescript KSII (+), respectively. Clone $\mathrm{pHI}-1: 3$ contained the $1 \cdot 5-\mathrm{kbp}$ Pst $\mathrm{I}-$ Pst I fragment (Fig. 2a). After incubation with the fluorogenic substrate, E. coli carrying the deletion derivative pHI-1:1 exhibited blue fluorescence with an intensity comparable with that observed for $\mathrm{pHI}-1$, whereas neither $\mathrm{pHI}-1: 2$ nor $\mathrm{pHI}-1: 3$ produced a fluorescence signal (Fig. 2a). These results suggest that ORF1, contained in the BamHI-HindIII fragment, conferred sialidase activity on the E. coli host.

In order to confirm that translation starts from the first ATG of ORF1, in vitro mutagenesis was performed. The region containing ORF1 was amplified by PCR with the primers R1, $\mathrm{R} 2$ and F1. The mean length of the PCR products of these primers was $1.4 \mathrm{kbp}$ (range $313-1710 \mathrm{bp}$ ). Sequencing confirmed that the R1 PCR product contained the wild-type ORF1 (initial ATG codon unchanged), whereas the R2 product contained a mutant ORF1 (first ATG codon changed to CGG). Transformant pBR1 (which contained the R1 PCR 
product) exhibited sialidase activity, whereas transformant pBR2 (which contained the R2 PCR product) did not (Fig. $2 \mathrm{~b})$. These results suggest that ORF1 of pHI-1 encodes T. forsythensis sialidase. We chose the name siaHI for the gene corresponding to ORF1.

\section{Nucleotide and predicted amino acid sequences of T. forsythensis ATCC $43037^{\top}$ sialidase}

Nucleotide sequence analysis of $\mathrm{pHI}-1$ : 1 revealed a potential translation initiation site at base position 313. An ORF of 1395 nucleotides (BamHI-HindIII fragment of pHI-1) would encode a protein of 465 amino acids, with a calculated molecular mass of $52 \mathrm{kDa}$ and a deduced pI of $6 \cdot 60$. A comparison of the properties of other bacterial sialidases with those of the siaHI product, even with PSI BLAST searches, suggested that the enzyme may represent a novel protein that has not previously been identified. For example, a similarity search of the EMBL and DDBJ databases using the FASTA and BLAST programs revealed that there was only $24 \%$ sequence identity (overlap of 379 amino acids) between the predicted amino acid sequences of T. forsythensis sialidase and ORF5 (oxidoreductase) of the Streptococcus pneumoniae neuraminidase gene (nanB; accession no. U43526). Moreover, the siaHI product exhibited no structural similarity to Asp box motifs (-Ser-X-Asp-X-Gly-X-Thr-Trp-) or RIP motifs (-Arg-Ile-Pro-), which are highly conserved among sialidases from phylogenetically unrelated organisms but not the active site of these enzymes (Roggentin et al., 1989). Therefore, it appears that the siaHI sialidase is not a classical one that has been reported previously but a novel protein unrelated to any known sialidases.

\section{Purification of $\boldsymbol{T}$. forsythensis sialidase}

As a first step towards purification, a cation-exchange column was employed to purify the sialidase from sonicated extracts from $T$. forsythensis ATCC $43037^{\mathrm{T}}$ and the sialidasepositive recombinant clone $\mathrm{pHI}-1: 1$. The major sialidase activity was detected in two fractions that eluted at $\mathrm{NaCl}$ concentrations of 150-250 mM in both samples (Fig. 3a, b). The concentrates were then subjected to an anion-exchange column. Active fractions having sialidase activity were eluted with the flow-through fraction in both samples (Fig. 3c, d) and were concentrated and $\mathrm{NaCl}$ was removed. In order to check the purity of these fractions, samples of $1 \mathrm{mg}$ (BSA equivalent) were analysed by SDS-PAGE $(7 \cdot 5-12 \cdot 0 \%)$. However, the purified samples stagnated in the stacking gel and did not migrate to the running gel; therefore, these samples were subjected to non-denaturing PAGE. The
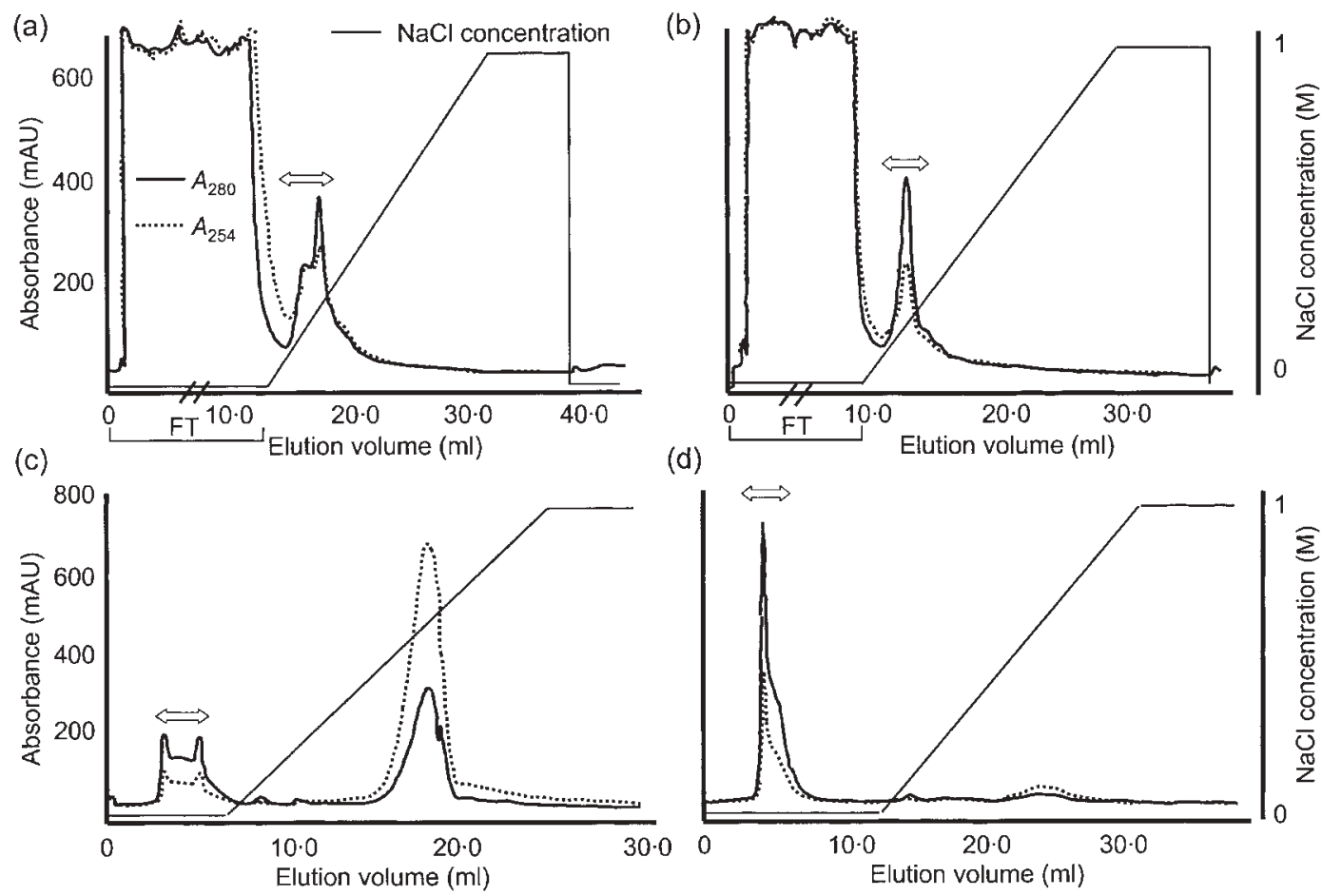

(d)

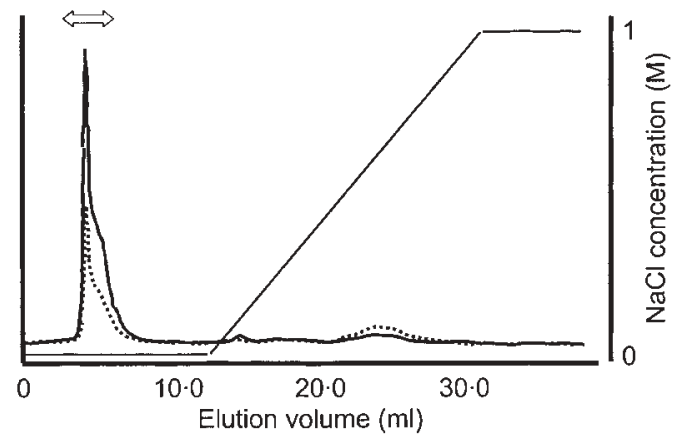

Fig. 3. (a) -(b) Fractionation of sialidase from T. forsythensis (a) and recombinant E. coli (pHI-1:1) (b) through a cation-exchange Resource S column. Sonicated extracts were loaded onto the column and flow-through (FT) and eluted adsorbates were assayed for sialidase. Sialidase activity was detected at 150-250 mM NaCl in both samples (double-headed arrows). (c)-(d) Fractionation of sialidase from T. forsythensis (c) and recombinant E. coli (pHI-1: 1) (d) through an anion-exchange Resource Q column. Sialidasepositive fractions from the Resource Q column were fractionated through the Resource S column. Sialidase activity was detected (double-headed arrows) in the flow-through fraction in both samples. 
enzymes from $T$. forsythensis ATCC $43037^{\mathrm{T}}$ and pHI-1:1 were respectively separated as two major bands and as a single band (Fig. 4a), and the sialidase activity of these protein bands was confirmed by sialidase assay using MUNeuNAc. As shown in Fig. 4(b), the purified protein preparation from pHI-1:1 showed sialidase activity and the size of this band was identical to that of the lower band from $T$. forsythensis. Furthermore, the upper band from T. forsythensis also demonstrated sialidase activity.

\section{Determination of the prevalence of the siaHI gene among clinical isolates of $T$. forsythensis}

In order to determine whether siaHI was present in clinically isolates of T. forsythensis, genomic DNA was prepared from three $T$. forsythensis isolates and subjected to hybridization at high stringency with a DNA probe representing the BbsIBbsI DNA fragment of pHI-1, a fragment (bases 165 to 2487) that contains the siaHI structural gene. This DNA probe hybridized strongly with a $2 \cdot 3$-kbp BbsI fragment of chromosomal DNA from T. forsythensis ATCC $43037^{\mathrm{T}}$ and the three clinical isolates (Fig. 5a). On the other hand, chromosomal DNA from E. coli HB101 containing plasmid pBluescript SKII $(+)$ or $(-)$ did not react with this probe in a separate experiment (data not shown). The three clinical isolates all exhibited sialidase activity using the filter-paper assay, but the presence of two distinct proteins was not determined. These results suggest that this sialidase gene is present in all three $T$. forsythensis clinical isolates (42-11, 2941 and 33-22).

\section{DISCUSSION}

The present communication describes the identification of the gene siaHI, encoding a sialidase, from $T$. forsythensis, which has been implicated as a major aetiological agent in periodontitis and a key pathogen for severe forms of chronic periodontitis. Sialidases have been demonstrated to function in a variety of host-associated activities that result in a modification of the host's ability to respond to bacterial infection (Holt \& Bramanti, 1991). Furthermore, the sialidase might be used by proteolytic bacteria to remove glycans from glycoproteins, thus rendering them more susceptible to proteolysis (Moncla et al., 1990). Sialidase production is also used to meet a nutritional requirement of the organism: without growth, there is usually no disease (Byers et al., 1999). T. forsythensis, similarly to Porphyromonas gingivalis, which is also strongly associated with periodontitis, has long been reported to produce sialidase (Moncla et al., 1990). However, whether sialidase production is involved in the pathogenesis of $T$. forsythensis and causes such periodontal disease remains to be clarified.

There were four potential ORFs in the sequence of the sialidase-encoding genomic DNA fragment, which differed (a)

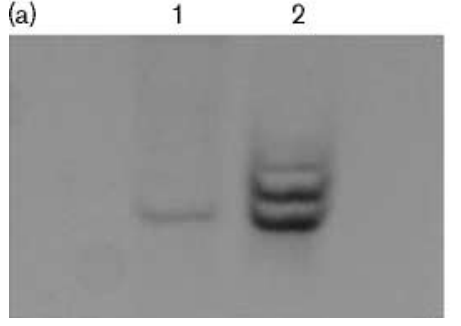

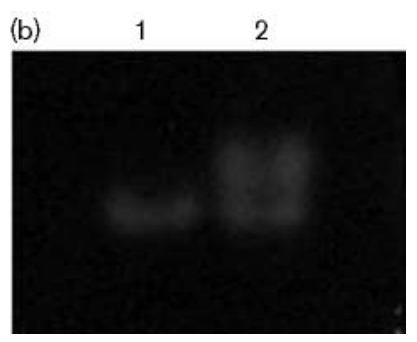

Fig. 4. Comparison of the molecular mass of purified enzyme from $T$. forsythensis and recombinant $E$. coli (pHI-1:1) by non-denaturing PAGE. Separate samples were fractionated by electrophoresis in a 10\% polyacrylamide nondenaturing gel with TG buffer (adjusted to $\mathrm{pH}$ 8.0 ) at $4{ }^{\circ} \mathrm{C}$ and visualized with Coomassie blue $\mathrm{R}-250$ protein staining (a) or stained for sialidase activity as outlined in Methods (b). Lanes: 1, purified fraction of a sialidase-positive $E$. coli clone (pHI-1:1); 2: purified sample of $T$. forsythensis ATCC $43037^{\top}$. (a)

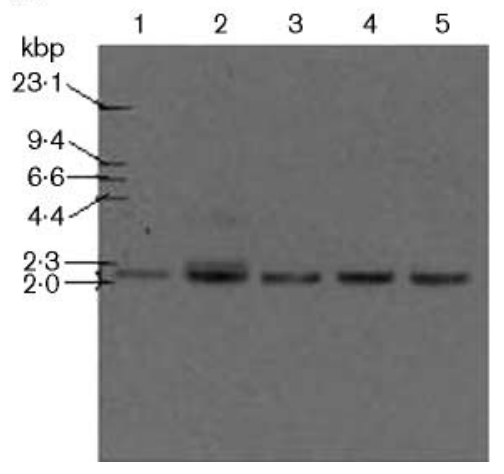

(b)

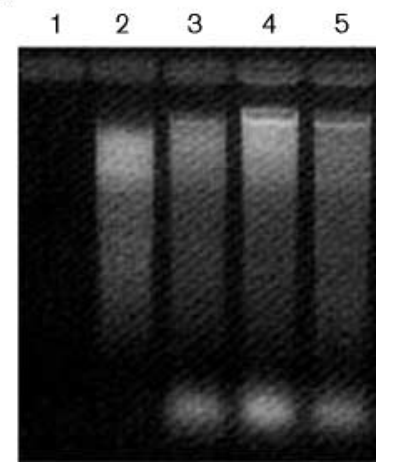

Fig. 5. Southern blot analysis. (a) DNA from $T$. forsythensis was digested with Bbsl, separated on $1.0 \%$ agarose gel and transferred to Hybond$\mathrm{N}+$ membrane. The 2-3-kbp Bbsl-Bbsl fragment of $\mathrm{pH}-1$ was labelled using an ECL labelling kit and used as the probe and hybridized bands were detected according to the manufacturer's instructions (Amersham). (b) Ethidiumbromide-stained gel. Lanes: 1, 2.3-kbp BbslBbsl fragment used as a probe; $2, T$. forsythensis ATCC $43037^{\top} ; 3-5$, three clinical isolates of $T$. forsythensis. 
in their orientations and lengths. The ORF of the sialidase gene was identified as follows. (i) Only pHI-1:1 of the deletion mutants that contained the four potential ORFs expressed sialidase activity. (ii) There was no other potential ORF that encoded a $52 \mathrm{kDa}$ protein in $\mathrm{pHI}-1$. (iii) A stop codon existed upstream from the ATG codon (at position 313) of pHI-1:1. (iv) Although there was no cluster that consisted of a ribosome-binding site and the corresponding ATG codon, protein was produced by this structural gene. In other words, this ORF was translated in E. coli. (v) Mutation of the corresponding ATG (Met) residue of this ORF led to loss of enzyme activity. These results suggested that the $1.4 \mathrm{kbp}$ sequence encoded sialidase. The overall $\mathrm{G}+\mathrm{C}$ content of ORF1 was $52.5 \mathrm{~mol} \%$, which is slightly higher than that of the T. forsythensis prtH genes (43.7 mol\%) (Saito et al., 1997). Since analysis of the $5^{\prime}$ end of this sequence revealed that there was a potential ribosome-binding site but no promoter-like sequences, it is possible that the siaHI gene is a part of an operon and is transcribed from a promoter further upstream. Ongoing DNA sequence studies and RNA transcriptional analyses should help to elucidate the transcriptional control of the siaHI gene.

The enzyme was purified from sonicated extracts of T. forsythensis ATCC $43037^{\mathrm{T}}$ and sialidase-positive clone pHI-1 : 1 by cation- and anion-exchange column chromatography. The enzymes from T. forsythensis ATCC $43037^{\mathrm{T}}$ and pHI-1: 1 were respectively separated as two major bands and as a single band (Fig. 4a) and the sialidase activity of these protein bands was confirmed by the sialidase assay using MUNeuNAc (Fig. 4b). Furthermore, as shown in Fig. 3, the enzymes from T. forsythensis and the E. coli clone (pHI-1:1) did not behave identically during chromatography (Fig. 3c, d). These results could be explained as follows: (i) modification of the enzyme might occur in T. forsythensis and not in E. coli, (ii) other sialidases might exist in this bacterium. Streptococcus pneumoniae expresses two distinct sialidases (neuraminidases), NanA (Berry et al., 1988), which is cellassociated, and NanB (Berry et al., 1996). These results revealed that the purified sample from pHI-1:1 might be identical to that from the original bacterium $T$. forsythensis. Obviously, further studies on the sizes of purified preparations using gel filtration, its location in the organism and the biological significance of $T$. forsythensis sialidase are required. Analysis of the crystal structure of purified sialidase should be carried out to elucidate the structure of this enzyme in nature and to elaborate selective potent inhibitors against the enzyme in a rational way.

In order to determine the prevalence and heterogeneity of the sialidase gene in this species, a flanking region of $\mathrm{pHI} 1: 1$ was detected in chromosomal DNA from clinical isolates by Southern blotting. Similar patterns of siaHI-hybridizing bands were obtained for all three clinical isolates of $T$. forsythensis from periodontitis patients. These results showed that siaHI is conserved among clinical isolates of $T$. forsythensis from periodontitis patients (all of which were found to produce sialidase; data not shown), suggesting that the sialidase encoded by siaHI could be a potential virulence factor in the pathogenesis of T. forsythensis in periodontal disease. Taken together, the available findings suggest that the sialidase produced by $T$. forsythensis strains, including clinical isolates from periodontitis patients, might be encoded by the novel sialidase gene siaHI.

The present results represent the first successful cloning of a sialidase gene (siaHI) from $T$. forsythensis. The evidence that we have cloned the structural sialidase gene consists of the observed enzyme expression in E. coli and the results of Southern blot analysis. A gene inactivation system has been developed for T. forsythensis (Honma et al., 2001) and isolation of the siaHI gene will make it possible to construct T. forsythensis mutants that are defective in this gene for testing in appropriate animal models. In this manner, it should be possible to define the existence of a second sialidase and the potential role of siaHI in the virulence of this periodontopathic bacterium. Clinically, the siaHI gene and the recombinant protein (SiaHI) could be utilized as a probe or antigen for detecting the virulence factor: sialidase or antisialidase antibody developed in patients from specimens such as subgingival plaque and saliva, and the correlation between the detection of this enzyme and clinical symptoms also should be evaluated.

\section{ACKNOWLEDGEMENTS}

This study was supported by a grant-in-aid from the Ministry of Education, Culture, Sports, Science, and Technology of Japan. Clinical isolates of T. forsythensis were provided by K. Yano (Tokyo Medical and Dental University, Graduate School, Department of Periodontology).

\section{REFERENCES}

Arakawa, S., Nakajima, T., Ishikura, H., Ichinose, S., Ishikawa, I. \& Tsuchida, N. (2000). Novel apoptosis-inducing activity in Bacteroides forsythus: a comparative study with three serotypes of Actinobacillus actinomycetemcomitans. Infect Immun 68, 4611-4615.

Berry, A. M., Paton, J. C., Glare, E. M., Hansman, D. \& Catcheside, D. E. A. (1988). Cloning and expression of the pneumococcal neuraminidase gene in Escherichia coli. Gene 71, 299-305.

Berry, A. M., Lock, R. A. \& Paton, J. C. (1996). Cloning and characterization of nanB, a second Streptococcus pneumoniae neuraminidase gene, and purification of the NanB enzyme from recombinant Escherichia coli. J Bacteriol 178, 4854-4860.

Bhown, A. S. \& Bennett, J. C. (1983). High-sensitivity sequence analysis of proteins recovered from sodium dodecyl sulfate gels. Methods Enzymol 91, 450-455.

Briselden, A. M., Moncla, B. J., Stevens, C. E. \& Hillier, S. L. (1992). Sialidases (neuraminidases) in bacterial vaginosis and bacterial vaginosis-associated microflora. J Clin Microbiol 30, 663-666.

Byers, H. L., Homer, K. A., Tarelli, E. \& Beighton, D. (1999). $\mathrm{N}$-Acetylneuraminic acid transport by Streptococcus oralis strain AR3. J Med Microbiol 48, 375-381.

Grossi, S. G., Zambon, J. J., Ho, A. W., Koch, G., Dunford, R. G., Machtei, E. E., Norderyd, O. M. \& Genco, R. J. (1994). Assessment of risk for periodontal disease. I. Risk indicators for attachment loss. J Periodontol 65, 260-267.

Grossi, S. G., Genco, R. J., Machtei, E. E., Ho, A. W., Koch, G., Dunford, 
R. G., Zambon, J. J \& Hausmann, E. (1995). Assessment of risk for periodontal disease. II. Risk indicators for alveolar bone loss. J Periodontol 66, 23-29.

Gyles, C. L. (1986). Erysipelothrix. In Pathogenesis of Bacterial Infections in Animals, p. 56. Edited by C. L. Gyles \& C. O. Thoen. Ames, IA: Iowa State University Press.

Holt, S. C. \& Bramanti, T. E. (1991). Factors in virulence expression and their role in periodontal disease pathogenesis. Crit Rev Oral Biol Med 2, 177-281.

Honma, K., Kuramitsu, H. K., Genco, R. J. \& Sharma, A. (2001). Development of a gene inactivation system for Bacteroides forsythus: construction and characterization of a BspA mutant. Infect Immun 69, 4686-4690.

Marmur, J. A. (1961). A procedure for isolation of deoxyribonucleic acid from microorganisms. J Mol Biol 3, 208-218.

McGregor, J. A., French, J. I., Richter, R., Franco-Buff, A., Johnson, A., Hillier, S., Judson, F. N. \& Todd, J. K. (1990). Antenatal microbiologic and maternal risk factors associated with prematurity. Am J Obstet Gynecol 163, 1465-1473.

Moncla, B. J. \& Braham, P. (1989). Detection of sialidase (neuraminidase) activity in Actinomyces species by using 2' -(4-methylumbelliferyl)- $\alpha$-D- $N$-acetylneuraminic acid in a filter paper spot test. J Clin Microbiol 27, 182-184.

Moncla, B. J., Braham, P. \& Hillier, S. L. (1990). Sialidase (neuraminidase) activity among Gram-negative anaerobic and capnophilic bacteria. J Clin Microbiol 28, 422-425.

Myers, R. W., Lee, R. T., Lee, Y. C., Thomas, G. H., Reynolds, L. W. \&
Uchida, Y. (1980). The synthesis of 4-methylumbelliferyl $\alpha$-ketoside of $\mathrm{N}$-acetylneuraminic acid and its use in a fluorometric assay for neuraminidase. Anal Biochem 101, 166-174.

Roggentin, P., Rothe, B., Kaper, J. B., Galen, J., Lawrisuk, L., Vimr, E. R. \& Schauer, R. (1989). Conserved sequences in bacterial and viral sialidases. Glycoconj J 6, 349-353.

Saito, T., Ishihara, K., Kato, T. \& Okuda, K. (1997). Cloning, expression, and sequencing of a protease gene from Bacteroides forsythus ATCC 43037 in Escherichia coli. Infect Immun 65, 4888-4891.

Sanger, F., Nicklen, S. \& Coulson, A. R. (1977). DNA sequencing with chain-terminating inhibitors. Proc Natl Acad Sci U S A 74, 5463-5467.

Sharma, A., Sojar, H. T., Glurich, I., Honma, K., Kuramitsu, H. K. \& Genco, R. J. (1998). Cloning, expression, and sequencing of a cell surface antigen containing a leucine-rich repeat motif from Bacteroides forsythus ATCC 43037. Infect Immun 66, 5703-5710.

Southern, E. M. (1975). Detection of specific sequences among DNA fragments separated by gel electrophoresis. J Mol Biol 98, 503-517.

Tanner, A. C., Strzempko, M. N., Belsky, C. A. \& McKinley, G. A. (1985). API ZYM and API An-Ident reactions of fastidious oral Gram-negative species. J Clin Microbiol 22, 333-335.

Tanner, A. C. R., Listgarten, M. A., Ebersole, J. L. \& Strzempko, M. N. (1986). Bacteroides forsythus sp. nov., a slow-growing, fusiform Bacteroides sp. from the human oral cavity. Int J Syst Bacteriol 36, 213-221.

Vimr, E. R. \& Troy, F. A. (1985). Regulation of sialic acid metabolism in Escherichia coli: role of $\mathrm{N}$-acetylneuraminate pyruvate-lyase. J Bacteriol 164, 854-860. 